\title{
Histone demethylase PHF8 accelerates the progression of colorectal cancer and can be regulated by miR-488 in vitro
}

\author{
YAO LV, YAN SHI, QUANLI HAN and GUANGHAI DAI \\ Department of Oncology, Chinese PLA General Hospital, Beijing 100853, P.R. China
}

Received June 18, 2016; Accepted April 19, 2017

DOI: $10.3892 / \mathrm{mmr} .2017 .7130$

\begin{abstract}
Plant homeo domain finger protein 8 (PHF8), as an oncogene, has been highlighted in cancer development and progression. However, its clinical significance and underlying molecular mechanisms in colorectal cancer (CRC) remain to be fully elucidated. In the present study, the role of PHF8 in the progression of CRC was investigated. The mRNA and protein levels of PHF8 in tissues from patients with CRC and cell lines were detected using the reverse transcription-quantitative polymerase chain reaction and western blotting, respectively. Cell viability was analyzed using an MTT assay. The targeted genes were predicted using a bioinformatics algorithm and confirmed by a dual luciferase reporter assay. Cell migration was evaluated using a Transwell assay. The results demonstrated that the expression of PHF8 was significantly increased in tumor tissues from patients with CRC and was correlated with tumor-node-metastasis stage. In addition, it was found that overexpressed PHF8 was a predictor of poor overall survival rates in patients with CRC. PHF8 loss-of-function significantly inhibited proliferation and migration, and promoted apoptosis of CRC cells. In addition, bioinformatics methods demonstrated that PHF8 was a putative target of microRNA (miR)-488, and miR-488 was able to inhibit the expression of PHF8 in CRC cells. miR-488 loss-of-function showed increased proliferation and migration, and these effects were reversed by sh-PHF8 transfection in CRC cells. In vitro and in vivo experiments revealed that $\mathrm{PHF} 8$ accelerated cancer cell growth and migration, confirming the oncogenic role of PHF8 in human CRC. In conclusion, PHF8 and miR-488 may serve as CRC biomarkers for the prediction of clinical outcome and provide a target for the diagnosis and therapy of CRC.
\end{abstract}

Correspondence to: Dr Guanghai Dai, Department of Oncology, Chinese PLA General Hospital, 28 Fuxing Road, Haidian, Beijing 100853, P.R. China

E-mail: dai_gh06@aliyun.com

Key words: plant homeo domain finger protein 8, colorectal cancer, microRNA-488, histone demethylation

\section{Introduction}

Colorectal cancer (CRC) is one of the most common types of malignancy and is the third most common type of cancer worldwide (1). In China, it was estimated that $\sim 376,300$ cases were diagnosed, and 191,000 Chinese succumbed to CRC-associated mortality in 2015 (2). In previous decades, the clinical therapeutic strategies for CRC have progressed significantly, however, the overall survival rate of patients with CRC has shown little improvement. An important reason for this is that the molecular mechanisms remain to be fully elucidated. Therefore, the investigation of the underlying molecular mechanisms for targeted therapy of CRC is important.

Plant homeo domain finger protein 8 (PHF8) is a JmjC domain-containing protein, which, as a chromatin regulator, can alter histone modifications and is linked to gene activation and repression (3). PHF8 is important in fundamental physiological functions and the progression of various diseases. PHF8 promotes T-cell activation and proliferation, and is a link to inflammatory responses and adaptive immunity (4). Histone methylation modulated by PHF8 is essential for endothelial cell migration (5). PHF8 has also been identified as a positive regulator of survival in response to mitochondrial dysfunction across species (6). PHF8 has been reported to be involved in several types of cancer, including breast cancer (7), non-small cell lung cancer (NSCLC) (8), acute promyelocytic leukemia (9) and esophageal squamous cell carcinoma (10). Previous studies have demonstrated that aberrant epigenetic alteration of histone lysine demethylation is crucial in the development of CRC (11). PHF2 and Jumonji-domain containing 3 (JMJD3) histone demethylase, a tumor suppressor, are downregulated in colon cancer (12). In addition, JMJD2B, lysine-specific demethylase 1 and JMJD2A histone demethylase are overexpressed in CRC cells or tissues (13-15). However, to the best of our knowledge, there have been no reports on the association of PHF8 with the development and progression of CRC.

In the present study, the role of PHF8 in CRC was investigated, and it was found that PHF8 was overexpressed in CRC and was associated with the tumor-node-metastasis (TNM) stage. PHF8 as an oncogenic protein promoted CRC cell proliferation, migration and invasion abilities. These results demonstrated that microRNA (miR)-488 was able to regulate PHF8 in CRC cell lines. 


\section{Materials and methods}

Patient samples. A total of $60 \mathrm{CRC}$ tissue samples and paired corresponding adjacent non-tumor tissue samples were collected from the Department of Oncology, Chinese PLA General Hospital (Beijing, China) between September 2012 and January 2015. All collected tissue samples were immediately stored in liquid nitrogen until use. The human samples were obtained with written informed consent from all patients. The study was approved by the Ethics Committee of the Department of Oncology, Chinese PLA General Hospital.

Cell culture. The normal epithelial cell line (NCM460) and four CRC cell lines (HCT116, HT29, SW480 and SW620) were obtained from the Cell Resource Center, Shanghai Institutes for Biological Sciences (Shanghai, China) and were maintained in RPMI-1640 (Thermo Fisher Scientific, Inc., Waltham, MA, USA) supplemented with 10\% FBS (Thermo Fisher Scientific, Inc.) at $37^{\circ} \mathrm{C}$ in a humidified incubator (Thermo Fisher Scientific, Inc.) with a $5 \% \mathrm{CO}_{2}, 95 \%$ air atmosphere.

Cell viability detection using 3-(4,5-dimethylthiazol-2-yl)2,5-diphenyltetrazolium bromide (MTT). The proliferation of the CRC cells was monitored using an MTT Cell Proliferation/Viability Assay kit (R\&D Systems, Inc., Minneapolis, MN, USA) according to the manufacturer's protocol.

Transwell migration assay. The CRC HCT116 and SW480 cells were treated with trypsinization and resuspension in $5 \%$ FBS medium to achieve a density of $1 \times 10^{5}$ cells $/ \mathrm{ml}$. Transwell inserts (8-mm pore size; Corning, Corning, NY, USA) were placed in wells containing medium and $10 \%$ FBS. A $200 \mathrm{ml}$ volume of cell suspension medium with 5\% FBS was added to the upper chamber and incubated at $37^{\circ} \mathrm{C}$ with $5 \% \mathrm{CO}_{2}$ dioxide for $48 \mathrm{~h}$. Following incubation for the appropriate period, the upper side of the membrane was washed and wiped using cotton swabs. The cells on the lower membrane surface were fixed with methanol for $10 \mathrm{~min}$ and stained with $1 \%$ toluidine blue (wt/vol; prepared in phosphate-buffered saline), for $5 \mathrm{~min}$, and washed twice with phosphate-buffered saline. When the dye had dried, $100 \mathrm{ml}$ of $10 \%$ acetic acid was added to the upper chamber and vortexed for $10 \mathrm{~min}$, followed by transfer into 96 -well plates. The OD570 values were measured using a BioTek Synergy MX microplate reader (BioTek Instruments, Inc., Winooski, VT, USA).

Terminal deoxynucleotidyl transferase-mediated dUTP nick end labeling (TUNEL) assay. The induction of apoptosis was monitored using the TUNEL method. The TUNEL assay was performed in accordance with manufacturer's protocol of the TUNEL apoptosis kit (R\&D Systems, Inc.).

Lentivirus preparation and infection. Short hairpin (sh)-blank control $(\mathrm{CN})$ and specific sh-RNAs targeting PHF8 were purchased from Invitrogen; Thermo Fisher Scientific, Inc., and the corresponding sequences were cloned into the pSIREN-RetroQ plasmid (Addgene, Inc., Cambridge, MA, USA) for retrovirus production with 293T cells (American Type Culture Collection, Manassas, VA, USA). The shRNA targeting
PHF8 (shPHF8) had the following sequence: 5'-ACTATGTTG GTTCTGACAA-3'; sh-CN had the following sequence: 5'-CGC GAGTTAGCGCGTGCTA-3'. For transduction, the 293T cells were incubated with virus-containing supernatant in the presence of $8 \mathrm{mg} / \mathrm{ml}$ polybrene. After $48 \mathrm{~h}$, the infected cells were selected for with puromycin $(2 \mathrm{mg} / \mathrm{ml})$ at $37^{\circ} \mathrm{C}$, following which the clones were selected and cultured for further experiments.

Luciferase reporter gene activity assay. The 3'-untranslated region (UTR) of the PHF8 gene, containing the predicated target sites for miR-488, was purchased from GenePharma Co., Ltd., Shanghai, China, and obtained from online prediction software (miRanda-mirSVR; www.microrna.org). The fragment was inserted into the multiple cloning sites of the pMIR-REPORT luciferase microRNA expression reporter vector (Ambion; Thermo Fisher Scientific, Inc.). The CRC cells $\left(2 \times 10^{5}\right)$ were co-transfected with $100 \mathrm{ng}$ luciferase reporters containing the PHF8 3'-UTR and miR-488 mimics using Lipofectamine 2000 (Invitrogen; Thermo Fisher Scientific, Inc.) at $37^{\circ} \mathrm{C}$. The cell lysates were harvested $24 \mathrm{~h}$ post-transfection and luciferase activity was measured using a dual luciferase reporter assay kit (Beyotime Institute of Biotechnology, Haimen, China) according to the manufacturer's protocol.

Transfection of cells with miR-488 mimics and inhibitor. The FAM-modified 2'-OMe-oligonucleotides were chemically synthesized and purified using high-performance liquid chromatography (GenePharma Co., Ltd., Shanghai, China). The 2'-OMe-miR-488 mimics were composed of RNA duplexes with the following sequence: 5'-UUGAAAGGCUAUUUC UUGGUC-3'. The sequences of the 2'-OMe-miR-488 inhibitor and 2'-Ome-scramble oligonucleotides were as follows: 5'-GACCAAGAAAUAGCCUUUCAA-3' and 5'-CUAUCA AUCGGCGGAUCCUAU-3'. The cells were transfected using Lipofectamine 2000 (Invitrogen; Thermo Fisher Scientific, Inc.) at a final concentration of $100 \mathrm{nM}$. At $24 \mathrm{~h}$ post-transfection, the culture medium was replaced and the cells were harvested $24 \mathrm{~h}$ later for analysis.

RT-qPCR analysis. RNA extraction was performed using TRIzol according to the manufacturer's protocol (Invitrogen; Thermo Fisher Scientific, Inc.). The synthesis of cDNAs was performed by reverse transcription reactions with $4 \mu \mathrm{g}$ of total RNA using moloney murine leukemia virus reverse transcriptase (Invitrogen; Thermo Fisher Scientific, Inc.) with oligo dT (15) primers (Fermentas; Thermo Fisher Scientific, Inc.) as described by the manufacturer. The levels of miR-488 were quantified using the mirVana qRT-PCR miRNA detection kit (Ambion; Thermo Fisher Scientific, Inc.) in conjunction with RT-qPCR with SYBR Green. PCR reaction mixtures (final volume, $25 \mu \mathrm{l}$ ) were prepared, including $12.5 \mu \mathrm{l}$ SYBR Green Supermix (Bio-Rad Laboratories, Inc., Hercules, CA, USA), $2 \mu 1 \mathrm{cDNA}, 300 \mathrm{nM}$ each primer, and diethylpyrocarbonate-treated $\mathrm{H}_{2} \mathrm{O}$. The cycling conditions were $95^{\circ} \mathrm{C}$ for $5 \mathrm{~min}$, followed by 38 cycles of $95^{\circ} \mathrm{C}$ for $15 \mathrm{sec}, 58^{\circ} \mathrm{C}$ for $30 \mathrm{sec}$ and $72^{\circ} \mathrm{C}$ for $30 \mathrm{sec}$. Following the cycle reaction, the quantification cycle $(\mathrm{Cq})$ was determined and relative miR-488 levels were calculated based on the $\mathrm{Cq}$ values normalized to the levels of U6 in each sample (16). PCR was performed with the following primers: PHF8, forward 5'-GACATGTGCCAG 
A

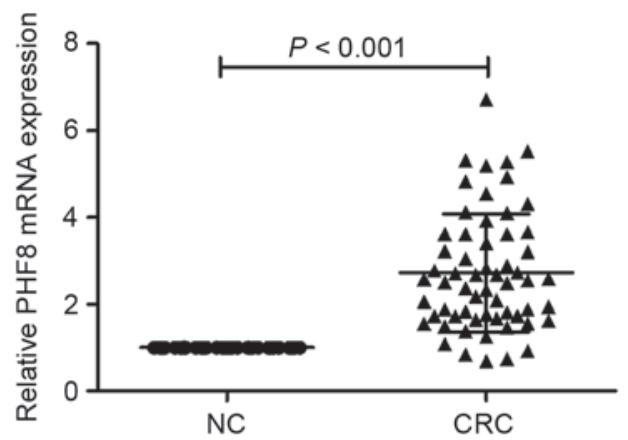

C

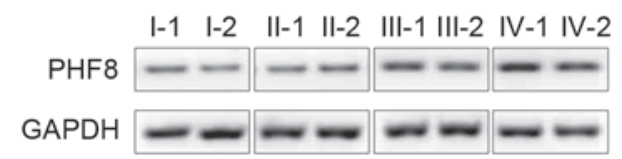

B

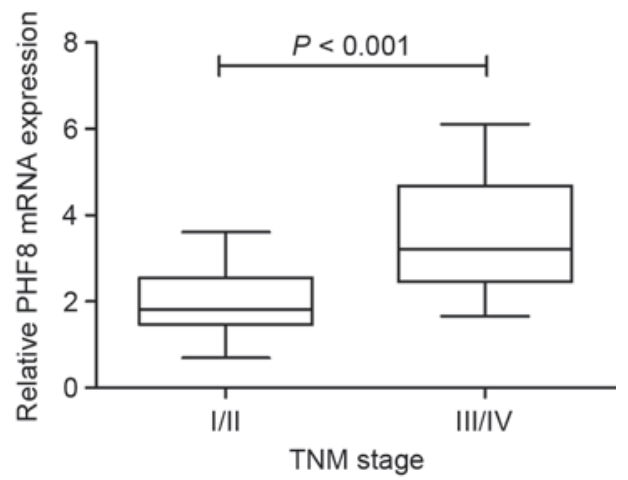

D

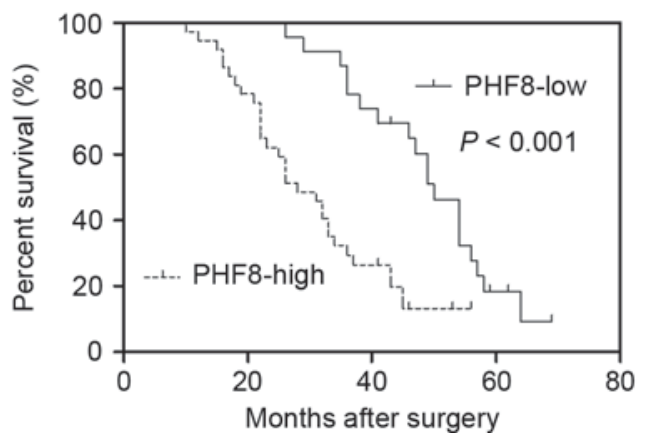

Figure 1. PHF8 is associated with a poor prognosis in patients with CRC. Expression of PHF8 in 60 colorectal cancer tissues and corresponding adjacent normal tissues. (A) mRNA expression of PHF8 was measured using reverse transcription-quantitative polymerase chain reaction analysis. (B) mRNA expression of PHF8 was measured in tissues of different TNM stages. (C) Protein expression of PHF8 was measured in tissues of different TNM stages. (D) Kaplan-Meier survival curve and log-rank test were used to evaluate whether the mRNA expression level of PHF8 was associated with overall survival rate. Values are expressed as the mean \pm standard deviation. $\mathrm{P}<0.05$ was considered to indicate a statistically significant difference. PHF8, plant homeo domain finger protein 8 ; TNM, tumor-node-metastasis; NC, non-cancerous.

GACTGGTTT-3' and reverse 5'-CAGCAGCCTTCTCCTCTT CAA-3'; GAPDH, forward 5'-GCACCGTCAAGCTGAGAA C-3' and reverse 5'-TGGTGAAGACGCCAGTGGA-3'.

Western blot analysis. The CRC tissues and cells were homogenized and extracted in NP-40 buffer. Protein concentrations were determined using the Bicinchoninic Acid kit for Protein Determination (cat. no. BCA1-1KT; Sigma-Aldrich; Merck KGaA, Darmstadt, Germany). Samples containing $50 \mu \mathrm{g}$ protein were separated on a $10 \%$ SDS-PAGE gel and transferred onto nitrocellulose membranes (Bio-Rad Laboratories, Inc.). Following saturation with $5 \%(\mathrm{w} / \mathrm{v})$ non-fat dry milk in TBS and $0.1 \%(\mathrm{w} / \mathrm{v})$ Tween $20(\mathrm{TBST})$, the membranes were incubated with the primary antibody anti-PHF8 (cat. no. ab191386; 1:2,000; Abcam, Cambridge, UK) at $4^{\circ} \mathrm{C}$ overnight. Following three washes with TBST, the membranes were incubated with the appropriate horseradish peroxidase-conjugated secondary antibody (cat. no. sc-516102; 1:20,000; Santa Cruz Biotechnology, Inc., Dallas, TX, USA) at room temperature for $1.5 \mathrm{~h}$, visualized using chemiluminescence (Thermo Fisher Scientific, Inc.) and analyzed using Quantity One software (version 4.4.0; Bio-Rad Laboratories, Inc.). GAPDH primary antibody (cat. no. 2118; 1:2,000; Cell Signaling Technology, Inc., Danvers, MA, USA) was used to as the control antibody.

Statistical analysis. The SPSS 15.0 software package (SPSS, Inc., Chicago, IL, USA) was used for general statistical analysis.
The significance of differences between groups was estimated using Student's t-test, one-way analysis of variance and $\chi^{2}$ test. Survival rates were calculated using the Kaplan-Meier method with a log-rank test applied for comparison. The correlation between level of PHF8 and levels of miR-488 was analyzed using linear regression analysis. $\mathrm{P}<0.05$ was considered to indicate a statistically significant difference.

\section{Results}

PHF8 is associated with a poor prognosis in patients with $C R C$. To determine whether PHF8 is involved in the progression of CRC, the present study first examined the mRNA and protein expression levels of PHF8 in cancer tissues and non-cancerous tissues from patients with CRC. The results of the RT-qPCR analysis showed that the mRNA expression of PHF8 was significantly increased in the cancer tissues, compared with that in the non-cancerous tissues (Fig. 1A). In addition, the mRNA expression of PHF8 was higher in samples of a high TNM grade, compared with those of a low TNM grade (Fig. 1B). It was also found that the protein expression of PHF8 was correlated with disease stage; the protein expression of PHF8 was markedly upregulated as TNM grade increased (Fig. 1C). To understand the prognostic significance of the upregulation of PHF8 in CRC, the association between the expression of PHF8 in CRC cancer tissues and patient survival rates was examined using the 
Table I. Clinicopathological characteristics and expression levels of PHF8 in patients with colorectal cancer.

\begin{tabular}{|c|c|c|c|c|}
\hline \multirow[b]{2}{*}{ Variable } & \multirow[b]{2}{*}{ Patients (n) } & \multicolumn{2}{|c|}{ Expression of PHF8 } & \multirow[b]{2}{*}{ P-value } \\
\hline & & Low (n) & High (n) & \\
\hline Age (years) & & & & 0.802 \\
\hline$<50$ & 19 & 7 & 12 & \\
\hline$\geq 50$ & 41 & 16 & 25 & \\
\hline Gender & & & & 0.625 \\
\hline Female & 24 & 8 & 16 & \\
\hline Male & 36 & 15 & 21 & \\
\hline TNM stage & & & & 0.005 \\
\hline $\mathrm{I} / \mathrm{II}$ & 23 & 15 & 8 & \\
\hline III/IV & 37 & 8 & 29 & \\
\hline $\begin{array}{l}\text { Lymph node } \\
\text { metastasis }\end{array}$ & & & & $<0.001$ \\
\hline Absent & 20 & 14 & 6 & \\
\hline Present & 40 & 9 & 31 & \\
\hline
\end{tabular}

PHF8, plant homeo domain finger protein 8; TNM, tumor-nodemetastasis.

Kaplan-Meier method, which revealed that overexpressed PHF8 predicted poor overall survival rates in the CRC patient cohort (Fig. 1D). Clinicopathological analysis indicated that PHF8 was significantly correlated with clinical stage and lymph nodes metastasis (Table I). These results suggested that PHF8 is involved in the progression of CRC.

PHF8 regulates proliferation, migration and apoptosis in CRC cell lines. To further investigate the potential role of PHF8 in the regulation of tumor progression, several CRC cell lines (HCT116, HT29, SW480 and SW620) and normal colorectal epithelial cells (NCM460) were examined. Consistent with the tumor tissue observations, the mRNA expression of PHF8 was upregulated in the HCT116, SW480 and SW620 CRC cell lines, compared with that in the NCM460 cell line (Fig. 2A). The protein expression of PHF8 was also upregulated in the HCT116 and SW480 CRC cell lines, compared with that in the NCM460 cell lines (Fig. 2B). Among the CRC cell lines, PHF8 was markedly overexpressed in the HCT116 and SW480 cell lines. Thus, the HCT116 and SW480 cell lines were used in subsequent experiments. The CRC cells were infected with a retrovirus carrying shPHF8, and the effects of PHF8 on proliferation, migration and apoptosis were evaluated. It was demonstrated that cell proliferation was markedly reduced when PHF8 was knocked down in the HCT116 and SW480 cell lines (Fig. 2C and D). A Boyden chamber was used to investigate the migration of CRC cell lines. As expected, the knockdown of PHF8 significantly inhibited HCT116 and SW480 cell migration (Fig. 2E). To investigate whether DNA damage was involved in the apoptosis induced by PHF8 knockdown, a TUNEL assay was performed. PHF8 loss-of-function significantly increased the number of TUNEL-positive cells in the HCT116 and SW480 cells (Fig. 2F). Taken together, these results indicated that PHF8 regulates proliferation, migration and apoptosis in CRC cell lines.

miR-488 is involved in the progression of CRC. The levels of miR-488 were measured in $60 \mathrm{CRC}$ tissue samples and non-cancerous tissues from patients with CRC. The results demonstrated that miR-488 was decreased in 50/60 (83.3\%) of the CRC tumor samples, and miR-488 was significantly downregulated in the cancer tissues, compared with the non-cancerous tissues (Fig. 3A). The expression of miR-488 was also significantly lower in tissues of a higher TNM grade, compared with that in tissues of a lower TNM grade (Fig. 3B). To understand the prognostic significance of the downregulation of miR-488 in CRC, the present study analyzed the association between miR-488 in CRC cancer tissues and patient survival rates using the Kaplan-Meier method. The downregulation of PHF8 predicted poor overall rates in the CRC cohort (Fig. 3C). Linear regression analysis was also performed to determine whether miR-488 was correlated with the expression of PHF8 in CRC. An inverse correlation was found between the expression levels of miR-488 and PHF8 in CRC tissues (Fig. 3D).

miR-488 targets the PHF8 3'-UTR in CRC cell lines. To further investigate the association between miR-488 and PHF8 in the present study, the MiRanda prediction algorithms were used. PHF8 was a putative target of miR-488, predicted by bioinformatics analysis (Fig. 4A). The bioinformatics analysis revealed that the PHF8 3'-UTR contained one conserved target site of miR-488. To confirm this, either the wild-type sequence of PHF8 or its mutant sequence were transfected into the pMIR luciferase reporter and then co-transfected with miR-488 or miR-NC into CRC cells. The relative luciferase activity of wild-type pmIR-PHF8 was significantly decreased by 55 and $50 \%$ in the HCT116 and SW480 cells, respectively (Fig. 4B). miR-488 mimics or inhibitors were also transfected into HCT116 or SW480 cells, and the expression of PHF8 in the HCT116 and SW480 cells was determined using RT-qPCR and western blot analyses. As expected, the miR-488 mimic-transfected HCT116 and SW480 cells exhibited lower mRNA and protein expression levels of PHF8 (Fig. 4C-F). By contrast, the miR-488 inhibitor-transfected HCT116 and SW480 cells exhibited higher mRNA and protein expression levels of PHF8 (Fig. 4C-F). The miR-488 inhibitor-transfected HCT116 and SW480 cells also exhibited increased proliferation, and this was reversed by sh-PHF8 transfection (Fig. 5A and B). The miR-488 inhibitor-transfected HCT116 and SW480 cells also exhibited increased cell migration, and this was also reversed by sh-PHF8 transfection (Fig. 5C and D).

\section{Discussion}

Previous studies have indicated that PHF8 may be an oncogene in certain types of cancer, including breast cancer (7) and NSCLC (8), however, the functional role and mechanistic action of PHF8 in CRC remain to be fully elucidated. In the present study, it was found that the expression of PHF8 was significantly increased in tumor tissues from patients with 

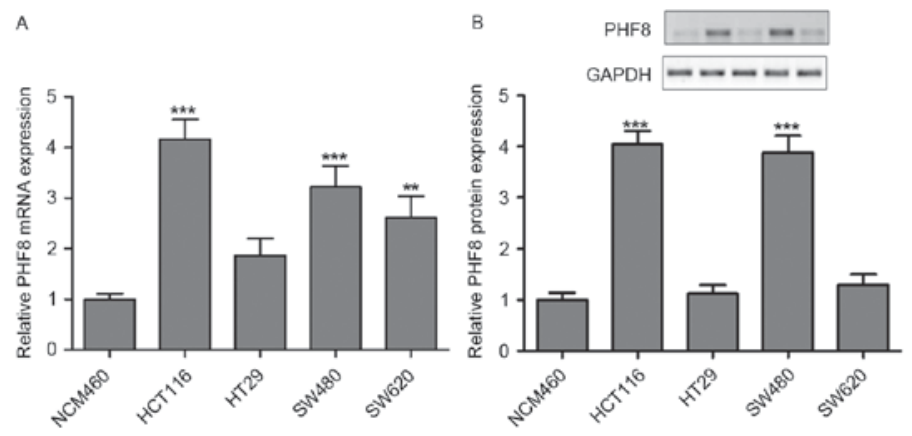

C
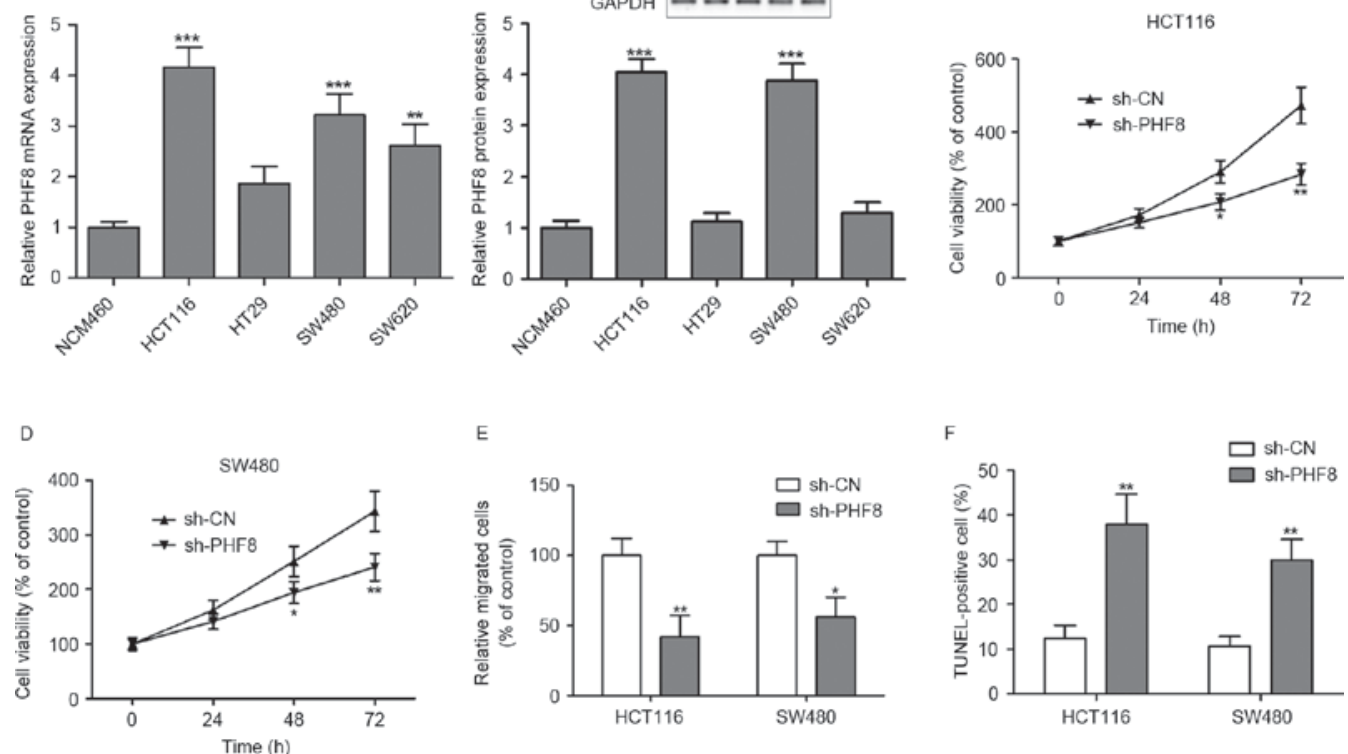

Figure 2. PHF8 regulates proliferation, migration and apoptosis in CRC cells. (A) mRNA and (B) protein expression of PHF8 were measured using reverse transcription-quantitative polymerase chain reaction and western bot analyses, respectively, in normal epithelial cell lines (NCM460) and four colorectal cancer cell lines (HCT116, HT29, SW480 and SW620). (C) HCT116 and (D) SW480 cells were infected with retrovirus carrying sh-CN or sh-PHF8, and cell viability was evaluated using a 3-(4,5-dimethylthiazol-2-yl)-2,5-diphenyltetrazolium bromide assay at 24, 48 and $72 \mathrm{~h}$ post-infection, respectively. (E) HCT116 and SW480 cells were infected with retrovirus carrying sh-CN or sh-PHF8, and cell migration was measured using a Transwell assay. (F) HCT116 and SW480 cells were infected with retrovirus carrying sh-CN or sh-PHF8, and apoptosis was measured using flow cytometric analysis. Values are expressed as the mean \pm standard deviation ( $\mathrm{n}=3$ in each group). ${ }^{*} \mathrm{P}<0.05,{ }^{* * *} \mathrm{P}<0.01$ and ${ }^{* * *} \mathrm{P}<0.001$, vs. control group. PHF8, plant homeo domain finger protein 8 ; sh-, short hairpin RNA; TUNEL, terminal deoxynucleotidyl transferase-mediated dUTP nick end labeling.

A

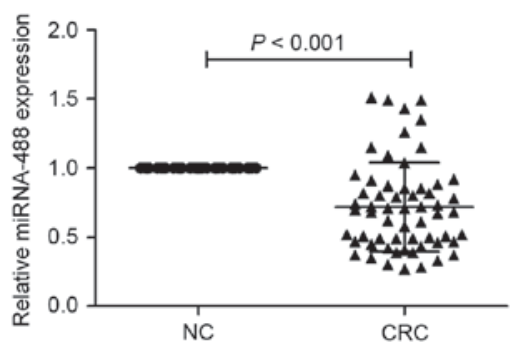

C

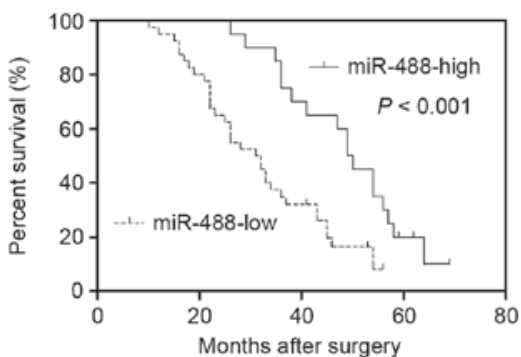

B

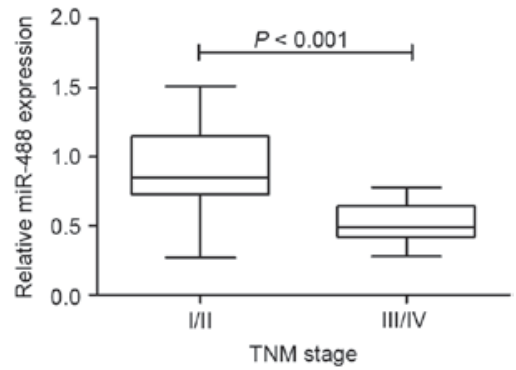

D

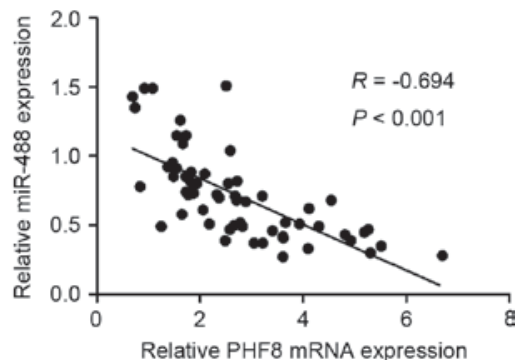

Figure 3. miR-488 is involved in the progression of CRC. (A) Expression of miR-488 in 60 CRC tissues and corresponding adjacent normal tissues, measured reverse transcription-quantitative polymerase chain reaction analysis. U6 was the endogenous control. (B) mRNA expression of miR-488 was measured in tissues at different TNM stages. (C) Kaplan-Meier survival curve and log-rank test were used to evaluate whether the expression level of miR-488 was associated with overall survival rate. (D) Correlation analysis was performed between mRNA expression levels of PHF8 and expression of miR-488 in CRC tissues. Values are expressed as the mean \pm standard deviation. $\mathrm{P}<0.05$ was considered to indicate a statistically significant difference. CRC, colorectal cancer; $\mathrm{NC}$, non-cancerous; PHF8, plant homeo domain finger protein 8; miR, microRNA; TNM, tumor-node-metastasis.

CRC. PHF8 was correlated with TNM stage, and it was found that the overexpression of $\mathrm{PHF} 8$ predicted poor overall survival rates in the $\mathrm{CRC}$ patient cohort. In vitro evidence showed that
PHF8 loss-of-function significantly inhibited proliferation and migration, and promoted apoptosis of HCT116 and SW480 cells. 


\section{3' cuGGUU-----CUUUAUCGGAAAGUu 5' hsa-miR-488

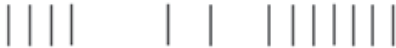 \\ 5' ugCCAACUUCACUUACCUUUCAa 3' PHF8 (wild)}

5' ugCCAACUUCACUUACCAAAGUa 3' PHF8 (mut)

C

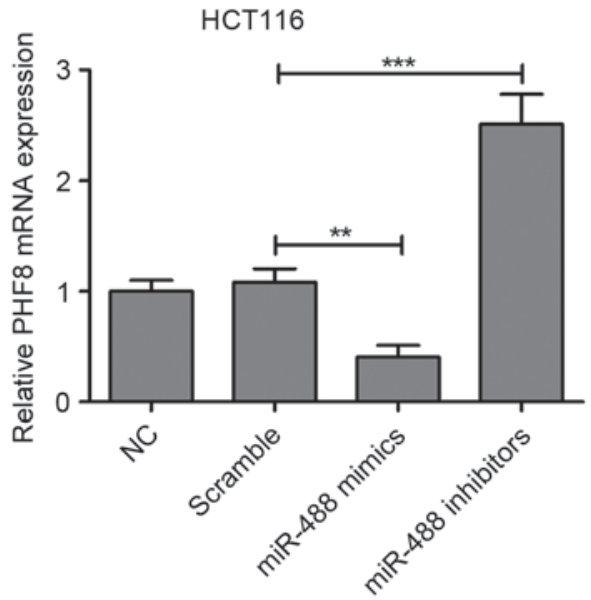

$\mathrm{E}$
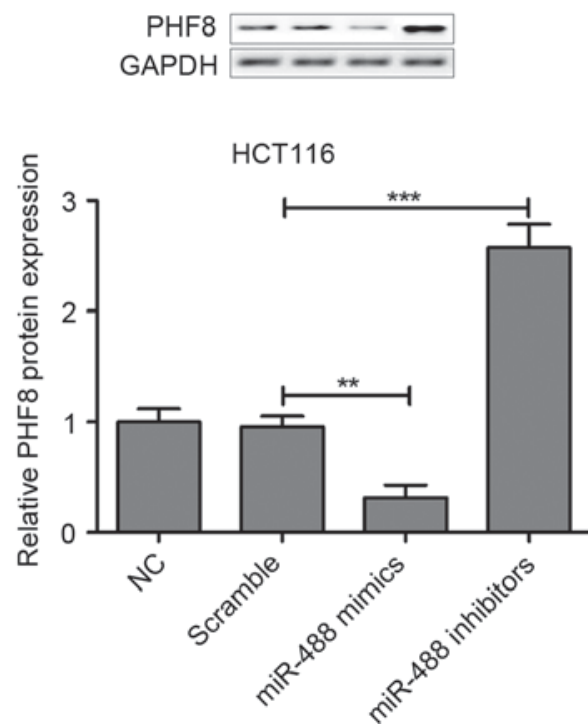

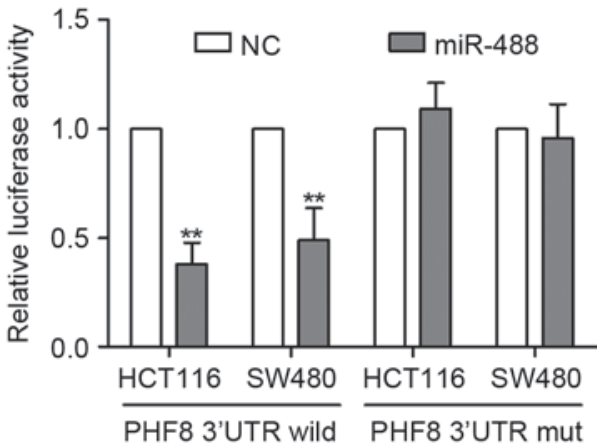

D

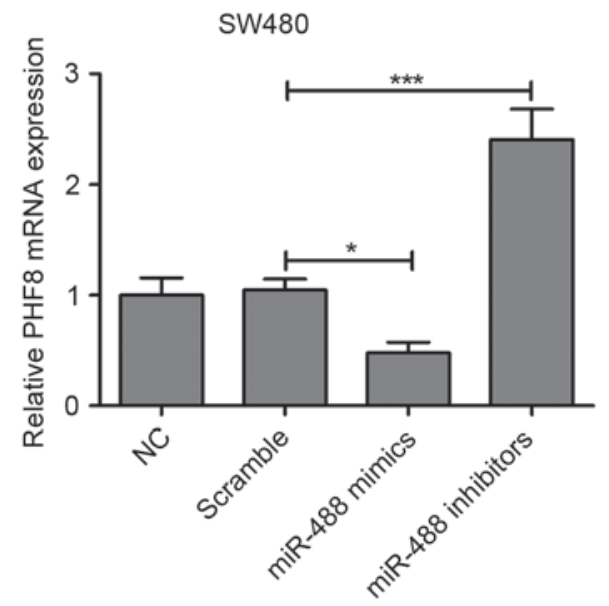

$\mathrm{F}$

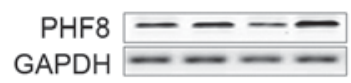

SW480

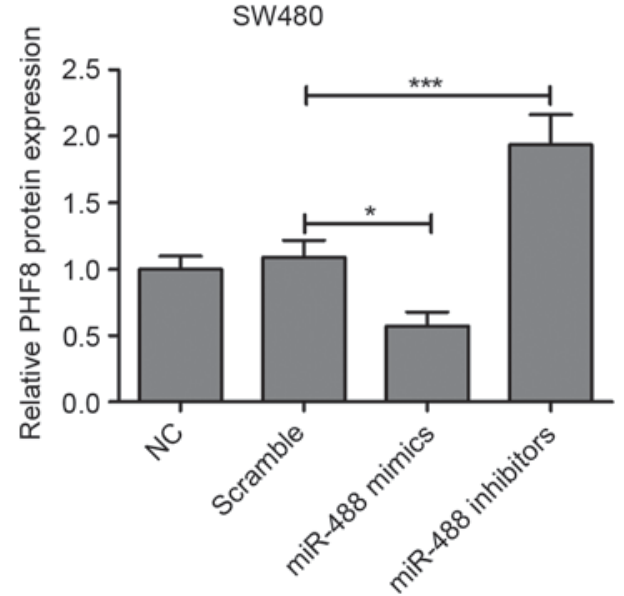

Figure 4. Base pairing between miR-488 and PHF8, predicted using bioinformatics analysis. (A) Schematic representation of the putative miR-488 binding site in the PHF8 3'-UTR. (B) Luciferase activity assay. Following transfection with miR-488 mimics or inhibitor for $48 \mathrm{~h}$, the mRNA expression of PHF8 was analyzed using reverse transcription-quantitative polymerase chain reaction analysis in (C) HCT116 and (D) SW480 cells. Following transfection, protein expression levels of PHF8 were analyzed using western blot analysis in (E) HCT116 and (F) SW480 cells. Values are expressed as the mean \pm standard deviation ( $\mathrm{n}=3$ in each group). ${ }^{*} \mathrm{P}<0.05,{ }^{* *} \mathrm{P}<0.01$ and ${ }^{* * * *} \mathrm{P}<0.001$, vs. control group. PHF8, plant homeo domain finger protein 8; NC, negative control; miR, microRNA; 3'-UTR, 3'-untranslated region; wild, wild-type; mut, mutant.

Histone modifications, including methylation, acetylation, phosphorylation and ubiquitination, are involved in a wide variety of biological and pathological processes, including cell differentiation, proliferation, apoptosis and metabolism (10). Among these histone modifications, methylation has attracted attention due to its central role in transcriptional regulation and other genomic functions (17). In the present study, the role of PHF8, a member of the most recently identified family of histone lysine demethylases, in the pathogenesis and progression of CRC was investigated. The results indicated that PHF8 
A

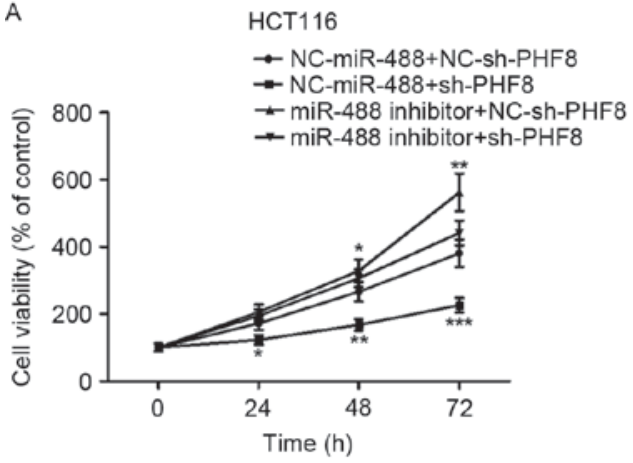

C

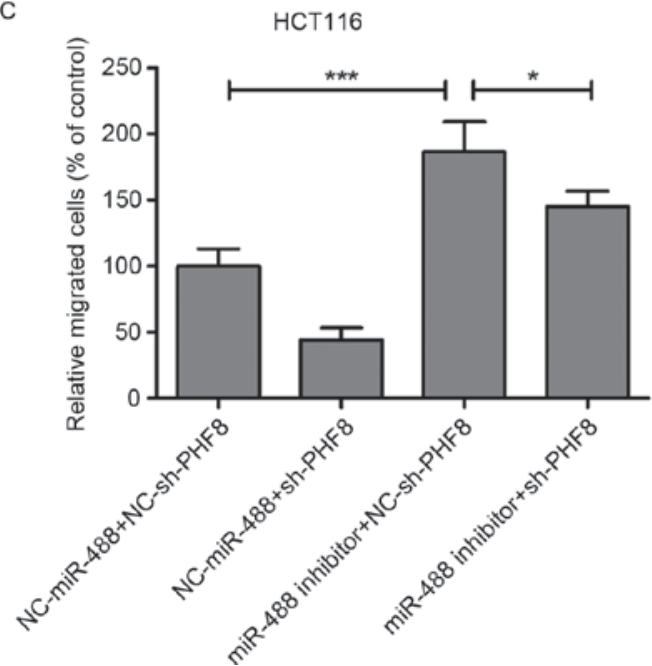

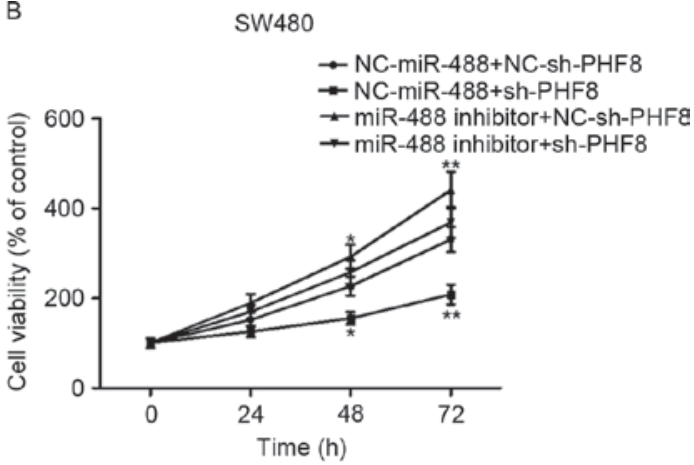

D

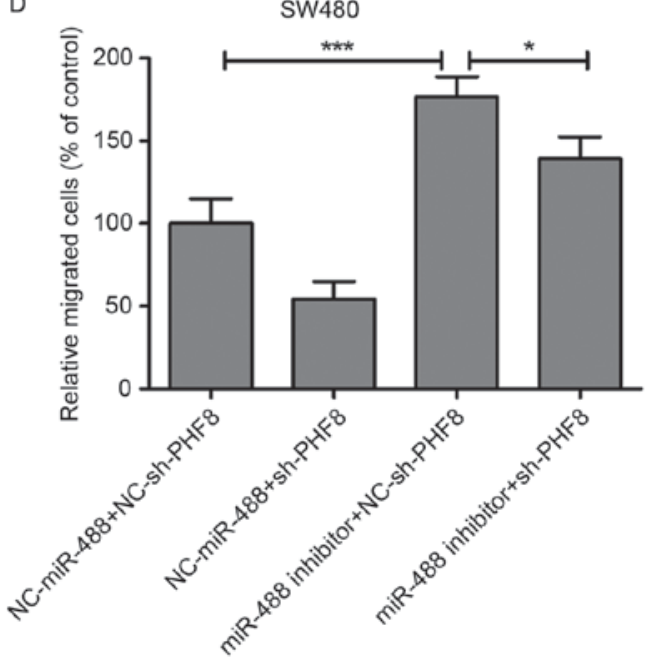

Figure 5. Effects of PHF8 on cell viability and migration. (A) HCT116 and (B) SW480 cells were infected with retrovirus carrying sh-PHF8 or miR-488 inhibitors, and cell viability was evaluated using a 3-(4,5-dimethylthiazol-2-yl)-2,5-diphenyltetrazolium bromide assay at 48 h post-infection. (C) HCT116 and (D) SW480 cells were infected with retrovirus carrying sh-PHF8 or miR-488 inhibitors, and cell migration was evaluated using a Transwell assay at $48 \mathrm{~h}$ post-infection. Values are expressed as the mean \pm standard deviation ( $\mathrm{n}=3$ in each group). ${ }^{*} \mathrm{P}<0.05,{ }^{* *} \mathrm{P}<0.01$ and ${ }^{* * * *} \mathrm{P}<0.001$, vs. control group. $\mathrm{PHF} 8$, plant homeo domain finger protein 8; sh-, short hairpin RNA; miR, microRNA; NC, negative control.

as an oncogene was involved in the progression of CRC. PHF8 has diverse roles in cellular activity and is recruited to promoters by its PHD domain, based on interactions with H3K4me2/3. It also controls $\mathrm{G} 1 / \mathrm{S}$ transition in conjunction with E2F1, HCF-1 and SET1A $(8,10)$. In breast cancer, PHF8 is overexpressed, and interaction with USP7 leads to the upregulation of cyclin A2 (7). The knockdown of PHF8 in ESCC cell lines has been shown to result in an inhibition of cell proliferation and an increase of apoptosis (10). In the present study, it was demonstrated that the knockdown of PHF8 inhibited cellular proliferation and migration, and an increased the apoptosis of HCT116 and SW480 cells. Consistent with previous reports, these results suggested that PHF8 as an oncogene regulated cellular proliferation and migration in cancer cells.

miRs are critical in the development and progression of human cancer through the post-transcriptional regulation of gene expression (10). Previous studies have indicated that miR-488 inhibits the proliferation and enhances the apoptosis of prostate carcinoma cells (18). miR-488 acts as a tumor suppressor gene, involved in the suppression of cell proliferation, cell cycle, colony information and migration in gastric cancer cells, and is negatively correlated with TNM stage in patients with gastric cancer (19). However, the functional role and the underlying molecular mechanisms of miR-488 in $\mathrm{CRC}$ remain to be fully elucidated. The results of the present study demonstrated that miR-488 was decreased in cancer tissues and was significantly lower in high TNM grade tissues, compared with that in low TNM grade tissues. In addition, an inverse correlation was found between the expression levels of miR-488 and PHF8 mRNA in CRC tissues. To further investigate the association between miR-488 and PHF8, bioinformatics methods demonstrated that PHF8 was a putative target of miR-488, and miR-488 inhibited the expression of PHF8 in CRC cell lines. miR-488 was involved in the progression of CRC; miR-488 loss-of-function resulted in increased proliferation and migration, which were reversed by sh-PHF8 transfection in HCT116 and SW480 cells. The in vitro and in vivo experiments revealed that PHF8 accelerated cancer cell growth and migration, confirming the oncogenic role of PHF8 in human CRC.

In conclusion, the data obtained in the present study support the carcinogenic role of PHF8 in CRC, and suggest that the PHF8 oncogene is involved in the progression of CRC. The preliminary findings indicated that PHF8, as a potential target of miR-488, can be suppressed by the overexpression of miR-488. Low levels of miR-488 and high levels of PHF8 were correlated with poor overall survival rates in patients with CRC. These results suggested that miR-488 and PHF8 may serve as CRC biomarkers for the prediction of clinical outcome and as targets for diagnosis and therapy in CRC. 


\section{Acknowledgements}

The present study was supported by the Project from Wujieping Medical Funding (grant. no. 320.6750.11076).

\section{References}

1. Zhao L, Yu H, Yi S, Peng X, Su P, Xiao Z, Liu R, Tang A, Li X, Liu F and Shen S: The tumor suppressor miR-138-5p targets PD-L1 in colorectal cancer. Oncotarget 7: 45370-45384, 2016.

2. Chen W, Zheng R, Baade PD, Zhang S, Zeng H, Bray F, Jemal A, Yu XQ and He J: Cancer statistics in China, 2015. CA Cancer J Clin 66: 115-132, 2016.

3. Liu W, Tanasa B, Tyurina OV, Zhou TY, Gassmann R, Liu WT, Ohgi KA, Benner C, Garcia-Bassets I, Aggarwal AK, et al: PHF8 mediates histone $\mathrm{H} 4$ lysine 20 demethylation events involved in cell cycle progression. Nature 466: 508-512, 2010.

4. Erdoğan O, Xie L, Wang L, Wu B, Kong Q, Wan Y and Chen X: Proteomic dissection of LPS-inducible, PHF8-dependent secretome reveals novel roles of PHF8 in TLR4-induced acute inflammation and T cell proliferation. Sci Rep 6: 24833, 2016.

5. Gu L, Hitzel J, Moll F, Kruse C, Malik RA, Preussner J, Looso M, Leisegang MS, Steinhilber D, Brandes RP and Fork C: The histone demethylase PHF8 is essential for endothelial cell migration. PLoS One 11: e0146645, 2016.

6. Merkwirth C, Jovaisaite V, Durieux J, Matilainen O, Jordan SD, Quiros PM, Steffen KK, Williams EG, Mouchiroud L, Tronnes SU, et al: Two conserved histone demethylases regulate mitochondrial stress-induced longevity. Cell 165: 1209-1223, 2016.

7. Wang Q, Ma S, Song N, Li X, Liu L, Yang S, Ding X, Shan L, Zhou X, Su D, et al: Stabilization of histone demethylase PHF8 by USP7 promotes breast carcinogenesis. J Clin Invest 126 2205-2220, 2016.

8. Shen Y, Pan X and Zhao H: The histone demethylase PHF8 is an oncogenic protein in human non-small cell lung cancer. Biochem Biophys Res Commun 451: 119-125, 2014.
9. Arteaga MF, Mikesch JH, Qiu J, Christensen J, Helin K, Kogan SC, Dong S and So CW: The histone demethylase PHF8 governs retinoic acid response in acute promyelocytic leukemia. Cancer Cell 23: 376-389, 2013

10. Sun X, Qiu JJ, Zhu S, Cao B, Sun L, Li S, Li P, Zhang S and Dong S: Oncogenic features of PHF8 histone demethylase in esophageal squamous cell carcinoma. PLoS One 8: e77353, 2013.

11. Tokunaga R, Sakamoto Y, Nakagawa S, Miyake K, Izumi D, Kosumi K, Taki K, Higashi T, Imamura Y, Ishimoto T, et al: The prognostic significance of histone lysine demethylase JMJD3/KDM6B in colorectal cancer. Ann Surg Oncol 23: 678-685, 2016.

12. Lee KH, Park JW, Sung HS, Choi YJ, Kim WH, Lee HS, Chung HJ, Shin HW, Cho CH, Kim TY, et al: PHF2 histone demethylase acts as a tumor suppressor in association with p53 in cancer. Oncogene 34: 2897-2909, 2015.

13. Fu L, Chen L, Yang J, Ye T, Chen Y and Fang J: HIF-1 $\alpha$-induced histone demethylase JMJD2B contributes to the malignant phenotype of colorectal cancer cells via an epigenetic mechanism. Carcinogenesis 33: 1664-1673, 2012.

14. Jie D, Zhongmin Z, Guoqing L, Sheng L, Yi Z, Jing W and Liang Z: Positive expression of LSD1 and negative expression of E-cadherin correlate with metastasis and poor prognosis of colon cancer. Dig Dis Sci 58: 1581-1589, 2013.

15. Kim TD, Shin S, Berry WL, Oh S and Janknecht R: The JMJD2A demethylase regulates apoptosis and proliferation in colon cancer cells. J Cell Biochem 113: 1368-1376, 2012.

16. Livak KJ and Schmittgen TD: Analysis of relative gene expression data using real-time quantitative PCR and the 2(-Delta Delta C(T)) method. Methods 25: 402-408, 2001.

17. Fang M, Ou J, Hutchinson L and Green MR: The BRAF oncoprotein functions through the transcriptional repressor MAFG to mediate the $\mathrm{CpG}$ island Methylator phenotype. Mol Cell 55: 904-915, 2014.

18. Sikand K, Slaibi JE, Singh R, Slane SD and Shukla GC: miR 488* inhibits androgen receptor expression in prostate carcinoma cells. Int J Cancer 129: 810-819, 2011.

19. Zhao Y, Lu G, Ke X, Lu X, Wang X, Li H, Ren M and He S: miR-488 acts as a tumor suppressor gene in gastric cancer. Tumour Biol 37: 8691-8698, 2016. 